Conclusion This analysis is the first to fit a chlamydia transmission model to national sex- and age- specific prevalence and case report time trends. The results suggest screening would have to achieve a higher coverage, or we should investigate novel strategies to reduce chlamydia prevalence further. This model could be used to investigate the impact of novel prevention interventions, such as improved partner notification strategies and targeted screening programs.

\section{Oral Presentation Session 2}

\section{Sexual Behaviour in Men Who Have Sex with Men}

\subsection{DIMENSIONALITY OF STIGMA AND ASSOCIATED ATTITUDES TOWARDS ANTICIPATED PARTNER NOTIFICATION AMONG MSM IN LIMA, PERU: AN EXPLORATORY FACTOR ANALYSIS}

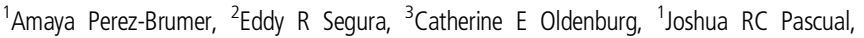
${ }^{4}$ Jorge Sanchez, ${ }^{4}$ Javier R Lama, ${ }^{5}$ Jesse L Clark. ${ }^{1}$ Columbia University Mailman School of Public Health, New York, USA; ${ }^{2}$ Department of Medicine, Division of Infectious Diseases, University of California, Los Angeles, USA; ${ }^{3}$ Francis I. Proctor Foundation, University of California, San Francisco, San Francisco, USA; ${ }^{4}$ Asociación Civil Impacta Salud Y Educación, Lima - Peru; ${ }^{5}$ Department of Medicine, Division of Infectious Diseases, University of California, Los Angeles, Los Angeles, USA
\end{abstract}

\section{$10.1136 /$ sextrans-2017-053264.6}

Introduction Partner notification (PN) is a key component of STI prevention efforts, yet, is underutilised by MSM, a population at-risk for STIs and HIV in Peru. To understand limiting mechanisms, we examined the dimensionality and latent factor structure of perceived STI and HIV stigma and attitudes towards PN among MSM in Lima, Peru.

Methods Between 2012-2014, 1,625 MSM in Lima were screened for HIV, syphilis, genital herpes and/or gonorrhoea/ chlamydia and completed a survey assessing sociodemographics, attitudes towards $\mathrm{PN}$, recent sexual practices, and STI and HIV-related stigma. Analytic plan included exploratory factor analysis (EFA) to assess dimensionality and interpretability of factor loadings on an item pool $(n=30)$ inquiring about anticipated $\mathrm{PN}$ and its perceived importance. All analyses conducted in MPlus v.7.

Results Participants (median age: 27, IQR: 23-34) reported median of 3 sexual partners (IQR: 1-5) and 64\% HIV seroprevalence. Of participants reporting sexual role: 334 (20\%) activo (top/insertive), 487 (30\%) pasivo (bottom/receptive), and 743 moderno (versatile). Eigenvalue analysis and EFA suggested a 3-factor model with simple structure best fit the observed covariance matrix (all loadings $>0.70$, 67\% of variability in data, CFI: 0.89, X2: 100690.

Conclusion Findings suggest the importance of underlying mechanisms linking STI-related enacted stigma and norms surrounding anticipated partner notification. Our results contribute to the existing knowledge on factors associated with anticipated PN in Peru and underscore the need for efforts to mitigate shame surrounding HIV and STI status, which may be important to improve acceptability and scale-up of PN and an HIV prevention interventions for MSM in Lima, Peru.
002.2 SYPHILIS AND SEXUAL GEOGRAPHIES: MAPPING THE SEXUAL TRAVELS, NETWORKS, AND KNOWLEDGE OF GAY, BISEXUAL AND OTHER MEN WHO HAVE SEX WITH MEN IN TORONTO, CANADA

'Dionne Gesink, '2James Connell, 'Lauren Kimura, 'Susan Wang, 'Daniel Grace, ${ }^{1}$ Adam Wynne, ${ }^{3}$ Sharmistha Mishra, ${ }^{3}$ Ann N Burchell, ${ }^{3}$ Darrell HS Tan, ${ }^{4}$ Travis Salway, ${ }^{5} J a s o n$ Wong, ${ }^{6}$ David Brennan, ${ }^{6}$ Carmen Logie, ${ }^{7}$ Mona Loutfy, ${ }^{5}$ Mark Gilbert. ${ }^{1}$ Dalla Lana School of Public Health, University of Toronto, Toronto, Canada; ${ }^{2}$ School of Population And Public Health, University of British Columbia, Vancouver, Canada; ${ }^{3}$ Li Ka Shing Knowledge Institute, St. Michael; ${ }^{4}$ University of Toronto, Toronto, Canada; ${ }^{5}$ British Columbia Centre for Disease Control, Vancouver, Canada; ${ }^{6}$ Factor-Inwentash Faculty of Social Work, University of Toronto, Toronto, Canada; ${ }^{7}$ Women's College Hospital, Toronto, Canada

10.1136/sextrans-2017-053264.7

Introduction A complex, persistent syphilis epidemic has affected gay, bisexual and other men who have sex with men (gbMSM) in major urban centres in North America for over a decade. Our objective was to explore the sexual travels, networks, and knowledge of gbMSM in Toronto.

Methods We conducted in-depth interviews between June and July 2016 with 31 gbMSM who were $\geq 18$ years, actively seeking sexual partners, and living, working or socialising in downtown Toronto. We asked participants to map their egocentric sexual network, share their sexual partner-seeking strategies and knowledge of STIs. We analysed travel patterns between participants and their sexual partners, including in relation to the core area of elevated syphilis rates, and used interview transcripts to interpret and explore the context from which observed patterns emerged.

Results Four geosexual archetypes dominated the egocentric sexual network maps, namely, hosters, two types of travellers: house-callers and rovers, and geoflexibles. These archetypes were observed in both core and noncore areas. Hosters usually or always hosted sex at their residence, creating a centralised sex phenomenon. Travellers rarely or never had sex at their residence. House-callers usually or always had sex at the residence of their sex partners; rovers also had sex at venues and other public spaces. Travellers created a dispersed sex phenomenon and bridging core, peripheral, and distant areas. Geoflexibles had sex anywhere (i.e.,home, venues, partner residences, public spaces). Participants practiced 1-2 online or inperson partner seeking strategies with little regard for syphilis because of overwhelming concern for HIV, lack of awareness of the syphilis epidemic, and treatability of the syphilis.

Conclusion Geo-sexual patterns and travel between sexual partners suggest specific archetypes exist comprising the larger gbMSM sociocentric sexual network. Future research should better define and characterise these archetypes and explore how each may impact STI transmission and intervention.

\subsection{UNDERSTANDING FUCKBUDDIES AMONG MEN WHO HAVE SEX WITH MEN}

Eric PF Chow, Vincent J Cornelisse, Clare Bellhouse, Jade E Bilardi, Sandra Walker, Christopher K Fairley. Melbourne Sexual Health Centre, Melbourne, Australia

\subsection{6/sextrans-2017-053264.8}

Introduction The term 'fuckbuddy' has been widely used in the men who have sex with men (MSM) population; however, this term is often classified as either 'regular' or 'casual' partnerships in sexual health research. We aimed to examine the 